\title{
A Traditional Chinese Herb Citri Reticulatae Pericarpium Induced Melanonychia
}

\author{
Shiyu Jiang \\ Jixiang $X u$ \\ Xia Xiong $\mathbb{D}$ \\ Yongqiong Deng (i)
}

Department of Dermatology \& STD, The Affiliated Hospital of Southwest Medical University, Luzhou, Sichuan, People's Republic of China
Correspondence: Yongqiong Deng Department of Dermatology \& STD, The Affiliated Hospital of Southwest Medical University, No. 25, Taiping Street, Luzhou, 646000 , Sichuan Province, People's Republic of China

Tel +86-|3679670608

Fax +86-830316565I

Email dengyongqiongl@I26.com
Abstract: The common causes for melanonychia include melanoma, repetitive trauma, underlying systemic diseases, onychomycosis, pseudomonas infection and drugs. Druginduced melanonychia usually affects numerous nails and appears as light brown to black pigmentation on the deck or nail bed with longitudinal, transverse or diffuse distribution. In previous cases, a number of chemotherapeutic agents such as azathioprine, bleomycin sulfate, cyclophosphamide, hydroxyurea and methotrexate were usually linked to melanonychia. Citri reticulatae pericarpium (CRP) is a traditional Chinese herb which is widely used in many foods and health care products in China. Up till now, there were no adverse reactions of CRP reported throughout the literature. Herein, we firstly reported a case of melanonychia in a 67-year-old man caused by CRP for external use.

Keywords: traditional Chinese herb, citri reticulatae pericarpium, melanonychia

\section{Introduction}

Melanonychia is the deposition of melanin in the nail plate, due to 2 different processes melanocytic activation or melanocytic hyperplasia. There are many and complex reasons for its formation and the common causes include melanoma, repetitive trauma, underlying systemic diseases, onychomycosis, pseudomonas infection and drugs ... Drug-induced melanonychia usually affects numerous nails and appears as multiple light brown to black longitudinal or transverse bands or diffuse nail discoloration, which depends on the type of melanocytic activation. A number of chemotherapeutic agents are associated with melanonychia, including bleomycin sulfate, cyclo-phosphamide, and methotrexate. ${ }^{1}$

Citri Reticulatae Pericarpium (CRP), a kind of traditional Chinese herb, is usually regarded to regulating qi, strengthening the spleen, drying dampness and resolving phlegm. Since thousands of years, CRP has been used widely in clinical practice to treat indigestion and inflammatory respiratory diseases such as bronchitis and asthma. In addition, CRP can be found in many foods and health care products in China. ${ }^{2}$ Up till now, there were no adverse reactions of CRP reported throughout the literature. This case firstly presented a 67-year-old man with melanonychia on all his toenails caused by CRP.

\section{Case Report}

A 67-year-old Chinese male presented with gradually progressive dark brown pigmentation on his toenails for about one month. There were no history of trauma, friction, or infection to his toenails. He was otherwise in good health and no history of any systemic 
medication could be recognized recently. Physical examination revealed that all the toenails were diffusely pigmented without discrete band appearance. The proximal nail folds from all the toenails were not affected and his fingernails were normal (Figure 1). The blackish pigmentation on the toenails could not be removed by alcohol cotton ball wiping or razor blade scratching. No pigmentation of the oral mucosa and/or any other abnormal pigmented lesion was found anywhere in the body. Microscopic examination of fungus showed negative. Laboratory tests such as tumor markers, blood routine, liver and kidney function were normal. Dermatoscopy showed homogeneous brown to black stain on all toenails without perinail skin involvement, longitudinal band and Hutchinson's sign. Moreover, histopathologic examination suggested that there were pigmentation granules on the deck without melanocyte proliferation (Figure 2).

For the health preservation of Traditional Chinese medicine, the patient soaked his feet in the solution of CRP for 10-15 minutes every night for half month. On the seventh day, light brown pigmentation was found on all the toenails and gradually it became dark brown. But the symptom was not obviously reduced after discontinuation of soaking for two weeks. After exclusion of melanonychia affecting multiple nails caused by repetitive trauma, subungual haematoma, onychomycosis, pseudomonas infection and underlying systemic disease, the diagnosis of drug-induced melanonychia due to external use of CRP solution was suggested for this patient. We did not give the patient any treatment, but only advised him to stop soaking with CRP solution. One month after discontinuation, the patient's toenails became pale and presented as scattered, uneven lightened brown pigmentation (Figure 3). Interestingly, his wife was not suffered from nail

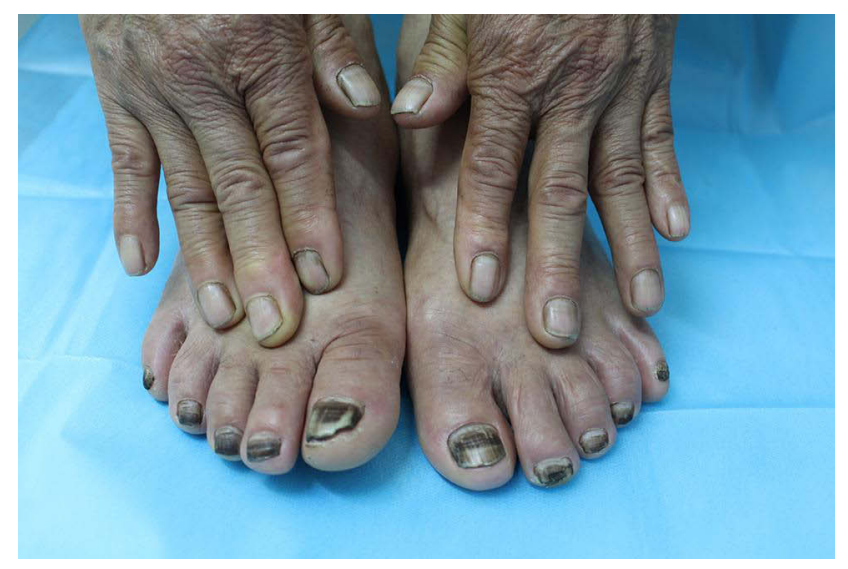

Figure I Clinical photograph showing diffusely blackish pigmentation on all toenails at the first visit.

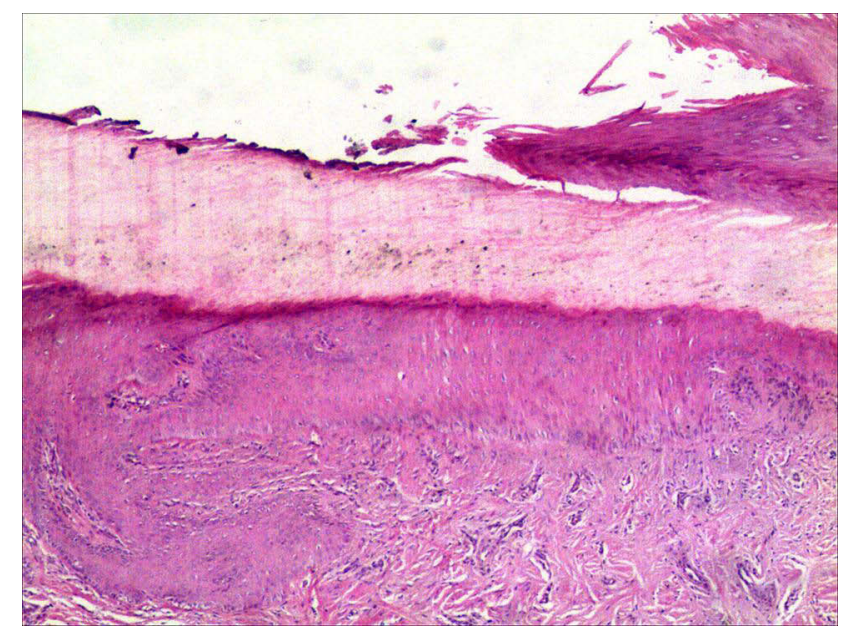

Figure 2 Histopathologic examination finding pigmentation granules on the deck (Hematox ylin-eosin stain; original magnification, $\times 10$.).

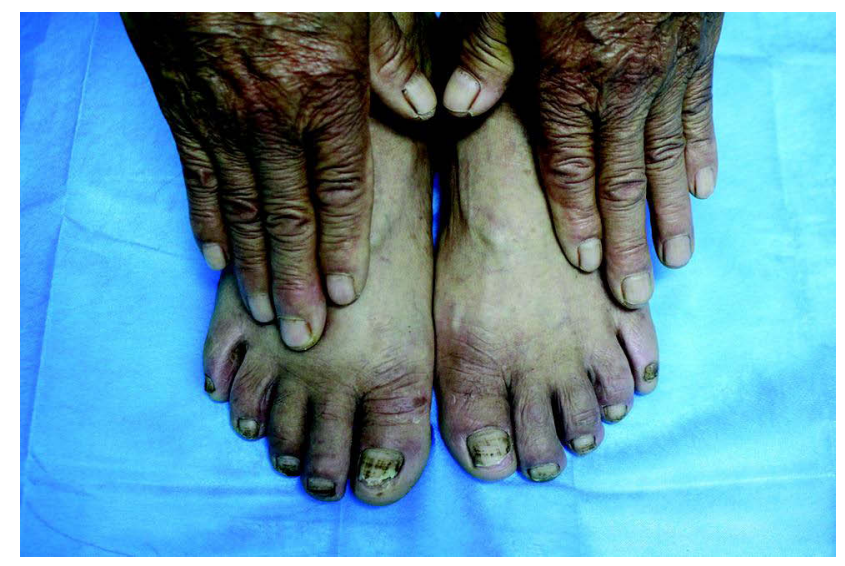

Figure 3 A month later, clinical photograph showing scattered lightened brown pigmentation on the toenails.

pigmentation who also soaked her feet in the same soup every night for half month.

\section{Discussion}

The characteristic of melanonychia is that the deck or nail bed is brown or dark brown, which can be distributed longitudinally, transversely or diffusely. The common pathogenesis of longitudinal melanonychia (LM) is increased melanocyte activity or melanocyte proliferation. ${ }^{1}$ When melanonychia is induced by factors such as drugs, racial, tobacco, dust, iodine, silver nitrate and pigmented nail lacquers, ${ }^{3}$ it usually appears diffuse, transverse and evenly distributed pigmentation. ${ }^{4}$ In this case, the characteristics of melanonychia contain multiple nails involved, diffuse and transverse distribution without discrete band appearance, short duration, history of 
external use of CRP solution and lightened pigmentation after discontinuation of this solution. According to the histopathological result of pigment granules in the deck, the diagnosis of drug-induced melanonychia due to external use of CRP solution could be made.

CRP is a traditional Chinese herb derived from the Citrus reticulata Blanco fruit peel. It has the functions of strengthening the spleen, relieving inappetence, lubricating the intestine, strengthening the bones, and enriching the kidney. ${ }^{5}$ It is also commonly added in many foods and health care products, which up till now has no adverse reactions recorded throughout the literature. This case for the first time reported a melanonychia caused by CRP for external use.

The color of CRP is mainly composed of naringin (a kind of flavonoids) and carotenoids. ${ }^{6,7}$ Generally, the pericarp contains natural pigment which can stain the surface of human skin, but the pigment can be disappeared after cleaning or scratching. In this case, the patient suffered blackish pigmentation on the toenails after using CRP solution which could not be removed after cleaning or scratching with alcohol cotton ball or razor blade. This phenomenon cannot simply be explained by exogenous staining of CRP and suggested the role of activation of melanocytes by CRP solution. But in a previous literature, South African scholars reported that ink (containing silver nitrate compound) can dye nails under ultraviolet light, and the ink was difficult to remove which can last for 2 4 weeks. ${ }^{8}$ However, the pigment was in the interior of the deck rather than on the surface by histological examination for the patient, which may be caused by the nail fold's reverse immersion from the lower deck. Recently, there was another case report of a child with longitudinal melanonychia caused by playing with green slime (a toy similar to clay), the slime entered the nail bed along the longitudinal ridge of the nail bed and the pigment disappeared spontaneously after two months. ${ }^{9}$ In this case, the patient's nail bed might be damaged, so it was more likely to be invaded by pigment. This also could explain why his wife was protected from melanonychia after soaking her feet in the CRP solution. At present, there is no special treatment for this disease, and the pigment can disappear with the growth of the toenail over time.

\section{Conclusion}

Drug-induced melanonychia was commonly associated with chemotherapeutic agents such as azathioprine, bleomycin sulfate, cyclophosphamide. Here we reported for the first time a case of melanonychia caused by a traditional Chinese herb CRP, which is widely used in many foods and health care products and with no adverse event reported throughout the literature. This case indicated a kind of side reaction melanonychia, which could be caused by CRP for external use, and has a self limited course after drug withdrawal.

\section{Consent for Publication and Ethics Approval}

The figures and patient's information in this manuscript were published with the patient's consent, and the patient had already read the manuscript and agreed with what was written. This article was performed in accordance with the principles of Declaration of Helsinki. Ethical review and institutional approval were not required for this case details.

\section{Disclosure}

The authors have no relevant affiliations or financial involvement with any organization or entity with a financial interest in or financial conflict with the subject matter or materials discussed in the manuscript. The authors report no conflicts of interest in this work.

\section{References}

1. Jefferson J, Rich P. Melanonychia. Dermatol Res Pract. 2012;2012:952186. doi:10.1155/2012/952186

2. Yu X, Sun S, Guo Y, et al. Citri Reticulatae Pericarpium (Chenpi): botany, ethnopharmacology, phytochemistry, and pharmacology of a frequently used traditional Chinese medicine. J Ethnopharmacol. 2018;220:265-282. doi:10.1016/j.jep.2018.03.031

3. Piraccini BM, Dika E, Fanti PA. Tips for diagnosis and treatment of nail pigmentation with practical algorithm. Dermatol Clin. 2015;33 (2):185-195. doi:10.1016/j.det.2014.12.002

4. Kim JH, Park JH, Lee DY. Site distribution of cutaneous melanoma in South Korea: a retrospective study at a single tertiary institution. Int J Dermatol. 2015;54(1):e38-9. doi:10.1111/ijd.12572

5. Piraccini BM, Alessandrini A, Starace M. Onychoscopy: dermoscopy of the Nails. Dermatol Clin. 2018;36(4):431-438. doi:10.1016/j. det.2018.05.010

6. Zheng G, Liu M, Chao Y, et al. Identification of lipophilic components in Citri Reticulatae Pericarpium cultivars by supercritical $\mathrm{CO} 2$ fluid extraction with ultra-high-performance liquid chromatography-Q Exactive Orbitrap tandem mass spectrometry. J Sep Sci. 2020;43(17):3421-3440. doi: $10.1002 /$ jssc. 202000490

7. Alós E, Cercós M, Rodrigo M, et al. Regulation of color break in citrus fruits. Changes in pigment profiling and gene expression induced by gibberellins and nitrate, two ripening retardants. $J$ Agric Food Chem. 2006;54(13):4888-4895. doi:10.1021/jf0606712

8. Prose NS. The Linear Nail Streak: a Lesson in Cultural Humility. JAMA Dermatol. 2017;153(10):1064-1065. doi:10.1001/jamade rmatol.2017.2328

9. Liang Y, Han XF, Ma L. Exogenous Pigmentation Presenting as Longitudinal Melanonychia in a Child. JAMA Dermatol. 2020;156 (1):101-102. doi:10.1001/jamadermatol.2019.3114 


\section{Publish your work in this journal}

Clinical, Cosmetic and Investigational Dermatology is an international, peer-reviewed, open access, online journal that focuses on the latest clinical and experimental research in all aspects of skin disease and cosmetic interventions. This journal is indexed on CAS.
The manuscript management system is completely online and includes a very quick and fair peer-review system, which is all easy to use. Visit http://www.dovepress.com/testimonials.php to read real quotes from published authors.

Submit your manuscript here: https://www.dovepress.com/clinical-cosmetic-and-investigational-dermatology-journal 